Figure 2 association of carbamylated anti-peptide antibodies with the presence of HLA DRB1 SE and non-SE in FDR

Anti-Ca-Fib2 in FDR with HL.A-DRB1 *1501

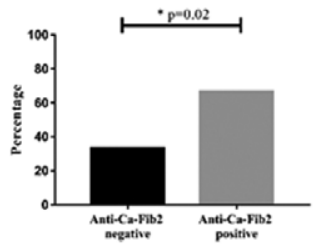

Anti-Ca-Fib2 in FDR with HLA-DRB1 *0407

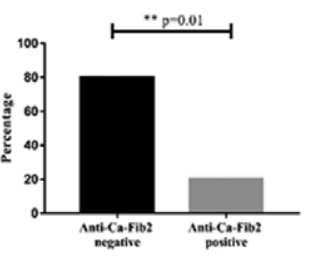

References:

[1] Shi J, van Steenbergen HW, van Nies JAB, et al. Arthritis Res Ther. $2015 ; 17(1)$

Acknowledgments: Hospital Militar Central (Grant 2015-047), the Government Institute of Science, Technology,and Innovation,Francisco Jose de Caldas-COLCIENCIAS(Grant No. 130865740792-2014)

Disclosure of Interests: None declared

DOI: 10.1136/annrheumdis-2020-eular.3861

\section{SAT0003 ELEVATED BASELINE AND INCREASING AUTOANTIBODY LEVELS ARE ASSOCIATED WITH INCREASED RISK FOR IMMINENT ONSET OF INFLAMMATORY ARTHRITIS IN A PROSPECTIVELY STUDIED ANTI-CITRULLINATED PROTEIN ANTIBODY POSITIVE COHORT: THE TIP-RA COLLECTIVE}

K. Deane ${ }^{1}$, G. Firestein ${ }^{2}$, D. Boyle ${ }^{2}$, J. Buckner ${ }^{3}$, E. A. James ${ }^{3}$, S. Posso ${ }^{3}$, W. Robinson ${ }^{4}$, L. K. Moss ${ }^{1}$, J. Seifert ${ }^{1}$, R. Gilmore ${ }^{1}$, S. Barzideh ${ }^{1}$, N. Rao ${ }^{5}$, F. Baribaud ${ }^{5}$, S. Nagpal ${ }^{5}$, A. Johnsen ${ }^{5}$, V. M. Holers ${ }^{1} .^{1}$ University of Colorado Denver, Aurora, United States of America; ${ }^{2}$ University of California San Diego, San Diego, United States of America; ${ }^{3}$ Benaroya Research Institute, Seattle, United States of America; ${ }^{4}$ Stanford University, Palo Alto, United States of America; Janssen Research and Development, LLC, Spring House, United States of America

Background: The Targeting Immune Responses for Prevention of RA (TIP-RA) Collaborative prospectively studies individuals at high risk for developing RA because of serum ACPA positivity in absence of baseline inflammatory arthritis (IA). Objectives: The objective of the analyses presented herein is to evaluate the role of baseline and changing levels of ACPA and rheumatoid factor (RF) in relationship to incident IA/RA.

Methods: ACPA+ subjects and ACPA- controls were identified who did not have baseline historical or examination evidence of IA. ACPA+ was defined by serum elevation of anti-CCP3 $\geq 20$ units (Inova). Subjects were evaluated annually or sooner if they had changes in joint symptoms. Factors including RFIgM and RFIgA (Inova) were also assessed, and relationships between autoantibody levels at baseline and over time and incident IA/RA were evaluated using t-tests, with paired testing where applicable.

Results: Baseline characteristics of ACPA+ and ACPA- subjects are in Table 1. Sixteen of the $94(17 \%)$ ACPA+ subjects developed IA/RA a mean of 518 days from the baseline visit; 14 of these met 2010 ACR/EULAR criteria for RA at the time of detection of IA. There was a trend for ACPA+ subjects who later developed IA/RA to have higher baseline levels of anti-CCP3 compared to those who did not develop IA/RA (Table 2). In addition, those who developed IA/RA had significantly higher mean levels of RFIgM and RFIgA compared to those who did not. While not statistically significant, in longitudinal analyses in the ACPA+ subjects with incident IA/RA, anti-CCP3 levels increased from baseline to identification of IA (mean [SD] of 119 [102] to 126 [100], $p=0.42$ ). Furthermore, RFIgM levels increased from $36[49]$ at baseline to 43 [51] at the time of $I A(p=0.31)$, and RFlgA levels increased from 16 [29] to 21 [31] $(p=0.10)$. In contrast, in ACPA+ subjects who did not develop IA/RA, anti-CCP3 levels increased only slightly over follow-up of a mean of 712 days: 75 [75] to 80 [76], $p=0.70$ while the levels of RFIgM and RFIgA decreased slightly during the same follow-up: for RFIgM mean [SD] levels went from 9 [22] to 8 [19], $p=0.74$; for RFIgA, 5 [16] to 3 [12], $p=0.67$.

Table 1. Baseline characteristics of ACPA+/- subjects

\begin{tabular}{lccc}
\hline & $\begin{array}{c}\text { ACPA- } \\
(\mathbf{n}=\mathbf{1 6 2})\end{array}$ & $\begin{array}{c}\text { ACPA+ } \\
(\mathrm{n}=94)\end{array}$ & p-value \\
\hline Age, mean & 58 & 58 & 0.90 \\
\% Female & 69 & 68 & 0.67 \\
\% Ever smoker & 33 & 34 & 0.87 \\
RF-IgM, mean (SD) & $3.2(10.0)$ & $13.5(30.2)$ & $<0.01$ \\
RF-IgA, mean (SD) & $0.3(0.6)$ & $6.5(19.1)$ & $<0.01$ \\
\hline
\end{tabular}

Table 2. Baseline characteristics of 16 ACPA+ subjects who developed incident IA/RA vs. 78 ACPA+ who did not

\begin{tabular}{lccc}
\hline & $\begin{array}{c}\text { Did not develop IA/RA } \\
(\mathrm{n}=78)\end{array}$ & $\begin{array}{c}\text { Developed IA/RA } \\
(\mathrm{n}=16)\end{array}$ & p-value \\
\hline $\begin{array}{l}\text { Days from baseline to IA/RA or } \\
\text { follow-up, mean (SD) }\end{array}$ & $712(124)$ & $518(295)$ & - \\
\% Meeting 2010 criteria at time of IA & - & & \\
CCP3, mean (SD) & $74.5(75.3)$ & $119.1(102.1)$ & 0.05 \\
RFIgM, mean (SD) & $9(22)$ & $36(49)$ & $<0.01$ \\
RFIgA, mean (SD) & $4(16)$ & $16(29)$ & $\mathbf{0 . 0 3}$ \\
\hline
\end{tabular}

Conclusion: In this prospectively followed cohort of ACPA+ subjects, higher levels of RFIgM and RFIgA at baseline were significantly associated with development of IA/RA within the follow-up period. Furthermore, there was a trend for rising levels of anti-CCP3 and RFIgM and A to be associated with development of IA/RA. These finding support the use of higher and/or rising levels of autoantibodies as additional features to predict imminent onset of IA/RA in ACPA+ individuals as well as potentially to use as outcomes of success of preventive interventions. Furthermore, the trend of increasing levels of RFIgM and RFIgA over time in individuals who developed IA/RA suggests that targeting pathways of RF development may lead to preventive interventions in a subset of RA.

References: None

Disclosure of Interests: Kevin Deane Grant/research support from: Janssen, Consultant of: Inova, ThermoFisher, Janseen, BMS and Microdrop, Gary Firestein Grant/research support from: Lilly, Janssen, Abbvie, David Boyle: None declared, Jane Buckner Grant/research support from: Bristol-Myers Squibb, Janssen, Eddie A. James Grant/research support from: Janssen, Pfizer, Sanofi, Novartis, Sylvia Posso Grant/research support from: Janssen, William Robinson Grant/research support from: Janssen, Laurie K. Moss Grant/research support from: Janssen, Jennifer Seifert Grant/research support from: Janssen, Roge Gilmore Grant/research support from: Janssen, Saman Barzideh Grant/research support from: Janssen, Navin Rao Shareholder of: Janssen Pharmaceuticals, Employee of: Janssen Pharmaceuticals, Frederic Baribaud Shareholder of: Janssen Research \& Development, LLC, Employee of: Janssen Research \& Development, LLC, Sunil Nagpal Shareholder of: Janssen Pharmaceuticals, Employee of: Janssen Pharmaceuticals, Alyssa Johnsen Employee of: Janssen, V. Michael Holers Grant/research support from: Janssen, Celgene, and BMS DOI: 10.1136/annrheumdis-2020-eular.5713

\section{SAT0004 \\ INCREASED M1 INFLAMMATORY PHENOTYPE OF CIRCULATING MONOCYTES IS ASSOCIATED WITH HISTORY OF CARDIOVASCULAR EVENTS IN RA PATIENTS}

A. Goecke ${ }^{1}$, C. Karsulovic ${ }^{1}$, J. Guerrero ${ }^{2}$, F. Tempio ${ }^{3}$, M. Lopez ${ }^{3} .{ }^{1}$ University of Chile Clinical Hospital, Rheumatology, Santiago, Chile; ${ }^{2}$ Faculty of Medicine, University of Chile, Physiology, Santiago, Chile; ${ }^{3}$ Faculty of Medicine, University of Chile, Immunology, Santiago, Chile

Background: Cardiovascular (CV) Disease is the main cause of death in Rheumatoid Arthritis (RA). Current tools like Framingham or European SCORE underestimate $\mathrm{CV}$ risk in RA patients. Efforts to improve the assessment including RA biomarkers (disease activity) have been only partially successful. There is a need for better biomarkers to identify AR patients at high risk for CV disease. Monocytes have an important role in plaque development. Monocytes differentiates into 2 main phenotypes M1 and M2 (1). In RA and in post-MI patients M1 monocytes are expanded (2). mTORC influences monocyte phenotype in vitro and has been associated with development of atheromatous plaque (3).

Objectives: To evaluate the phenotype of circulating monocyte in RA patient with or without previous CV events (RA-CV(-)RA-CV $(+))$, and its possible association with $\mathrm{mTORC}$ activity.

Methods: 9 RA-CV(+)patients aged between 18 and 65 yo with RA (EULAR/ ACR 2010 criteria), were paired with RA-CV(-)patients. 6 healthy individuals (HI) were also studied. Pairing criteria were classic CV risk factors (AHA 2018), sex, age, years since RA diagnosis, comorbidities, number of DMARDs previously used and use of bDMARDS. M1 and M2 circulating Monocytes were evaluated in PBMC obtained from patients and controls by flow cytometry analysis. Intracellular inflammatory cytokines (IL1, II6) and phosphorylated S6R (P-S6R) as a measure of mTORC activation was also evaluated. M1 was defined as CD14+HLA-DR+CCR2+ and M2 CD14+CD163+CCR2-. DAS28-RCP, DAS28-ESR and Lipid profile was also measured. The differences among groups was analysed using Mann-Whitney $U$ nonparametric. The relationship between variables with Spearman rank correlation test.

Results: There were no differences in demographic, RA characteristic and $\mathrm{CV}$ risk factors between RA-CV (+) and RA-CV (-) patients. Male/Female 4/5, age $62 \pm 3$ and $63 \pm 2$ respectively. $\mathrm{HI}$ were younger than RA patients $(32.5 \pm 7)$. CV events were 8 patients with $\mathrm{MI}$ and one Stroke. DAS28-RCP was $2.96 \pm 0.23$ and $2.88 \pm 0.43$ respectively. One patient in each group had failed to more than 2 sDMARDs 